Pacific Journal of Mathematics

INTRINSIC TOPOLOGIES IN TOPOLOGICAL LATTICES AND 


\title{
INTRINSIC TOPOLOGIES IN TOPOLOGICAL LATTICES AND SEMILATTICES
}

\author{
JIMMIE D. LAWSON
}

\begin{abstract}
This paper demonstrates that the topology of a compact topological lattice or semilattice can be defined intrinsically, i.e., in terms of the algebraic structure. Properties of various intrinsic topologies are explored.
\end{abstract}

A variety of ways have been suggested for defining topologies from the algebraic structure of a lattice (see e.g. [4] or [12]). If one is given a topological lattice, a natural question is whether the given topology agrees with one or more of these intrinsic topologies. Some results of this nature may be found in [5] or [13]. In this paper we show that the topology of a compact topological lattice or semilattice can always be defined intrinsically; these results extend to a large class of locally compact lattices.

A topological lattice is a lattice $L$ equipped with a Hausdorff topology for which the operations of join and meet are continuous as mappings from $L \times L$ into $L$. A topological semilattice is a (meet) semilattice together with a Hausdorff topology for which the meet operation is continuous.

If $A$ is a subset of a lattice or semilattice, we define

$$
L(A)=\{y: y \leqq x \text { for some } x \in A\}
$$

and

$$
M(A)=\{z: x \leqq z \text { for some } x \in A\} .
$$

A subset $B$ of a semilattice is an ideal if $L(B)=B$. A set $A$ is convex if $x, z \in A$ and $x \leqq y \leqq z$ imply $y \in A$. A lattice $L$ is locally convex if it has an open base of convex sets. A closed interval is a set of the form

$$
[a, b]=\{x: a \leqq x \leqq b\} \text {. }
$$

For the definition of undefined lattice properties employed in this paper, the reader is referred to [4].

The topological closure of a set $A$ will be denoted by $A^{*}$.

1. Intrinsic topologies. The following intrinsic topologies on a lattice $L$ are considered in this paper.

(1) The interval topology (I). If $L$ has a 0 and 1 , the interval topology is defined by taking as a subbase for the closed sets all sets 
$\{L(x): x \in L\}$ and all sets $\{M(x): x \in L\}$. If $L$ does not have universal bounds, then a set $K \subset L$ is closed if $K \cap[a, b]$ is closed in the interval topology of the sublattice $[a, b]$ for all $a, b$ with $a \leqq b$.

(2) The order topology (0). A net $\left\{x_{\alpha}\right\}$ in $L$ is said to orderconverge to $x$ if there exist a monotonic ascending net $\left\{t_{\alpha}\right\}$ with $x=$ $\sup t_{\alpha}\left(t_{\alpha} \uparrow x\right)$ and a monotonic descending net $\left\{u_{\alpha}\right\}$ with $x=\inf u_{\alpha}\left(u_{\alpha} \downarrow x\right)$ such that for all $\alpha, t_{\alpha} \leqq x_{\alpha} \leqq u_{\alpha}$. A subset $A$ of $L$ is closed in the order topology if $\left\{x_{\alpha}\right\} \subset A$ and $x_{\alpha}$ order converges to $x$ imply that $x \in A$. Note that if $x_{\alpha}$ order-converges to $x$, then for any cofinal subset of the domain directed set it remains true that $x_{\alpha}$ order-converges to $x$. Hence the order topology may be defined equivalently by declaring a set $U$ of $L$ open if $x \in U$ and $x_{\alpha}$ order-converges to $x$ imply $x_{\alpha}$ is residually in $U$.

(3) The convex-order topology (CO). A subset $U$ of $L$ is a basic open set for the convex-order topology if (i) $U$ is convex and (ii) if $x_{\alpha}$ order-converges to $x, x \in U$, then $x_{\alpha}$ is residually in $U$. Again, the second condition is equivalent to $U$ being open in the order topology.

We now list some easily derived properties of these intrinsic topologies.

Proposition 1. (1) The CO topology is locally convex.

(2) The 0 topology is finer than the CO topology.

(3) Any homomorphism from $L$ to a locally convex lattice that is continuous in the 0 topology is continuous in the $\mathrm{CO}$ topology.

(4) If the 0 topology is locally convex, then it agrees with the CO topology.

Proposition 2. The 0 topology is finer than the I topology.

Proof. [4, p. 251].

We shall call a topology on a lattice agreeable if (i) the topology is locally convex and (ii) if $t_{\alpha} \uparrow x$ or $t_{\alpha} \downarrow x$ then $t_{\alpha}$ converges to $x$ in the topology.

Proposition 3. If $\tau$ is an agreeable topology on a lattice $L$, then the CO topology is finer than $\tau$.

Proof. Since $\tau$ is locally convex, it suffices to show that if a convex set $U$ is in $\tau$, then it is open in the CO topology. Suppose that $x_{\alpha}$ is a net that order-converges to $x \in U$. Then there exist $t_{\alpha} \uparrow x, u_{\alpha} \downarrow x$ such that for all $\alpha, t_{\alpha} \leqq x_{\alpha} \leqq u_{\alpha}$. Since $\tau$ is agreeable, $t_{\alpha}$ and $u_{\alpha}$ are residually in $U$, and since $U$ is convex $x_{\alpha}$ is residually in $U$. 
2. The interval topology in complete lattices. The interval topology has received rather through investigation. In this section we summarize results concerning its relationship to compact topological lattices.

Proposition 4. Let $L$ be a complete lattice.

(1) $L$ is compact in the interval topology.

(2) If $(L, \tau)$ is a topological lattice, then $\tau$ is finer than the interval topology.

(3) If $L$ is Hausdorff in the interval topology, then the order and interval topology coincide.

Proof. (1) This is a result of O. Frink. A proof may be found $[4$, p. 250].

(2) Since in a topological lattice $M(x)$ and $L(x)$ are closed for each $x \in L$, and these sets are a subbasis for the closed sets of the interval topology, the result follows.

(3) See [3] or [15].

The next theorem contains the central results on compact topological lattices with the interval topology.

THEOREM 5. The following are equivalent in a compact topological lattice $(L, \tau)$ :

(1) $(L, I)$ is Hausdorff.

(2) $\tau=0=I=\mathrm{CO}$.

(3) $(L, \tau)$ has a basis of open convex sublattices.

(4) $(L, \tau)$ has a base of neighborhoods at each point of closed intervals.

(5) If $y \geqq x$ then there exists $z$ such that $x$ is in the interior of $L(z)$ and $y \not z$, and dually.

(6) Every net has an order-convergent subnet.

Proof. The equivalence of $3,4,5$ has been shown by E. B. Davies [6, Theorem 5]. K. Atsumi has shown the equivalence of 1 and 6 [3, Theorem 3]. D. Strauss has shown the equivalence of 1 and 3 [13, Theorem 5]. Conditions 3 and 1 together with part 2 of Proposition 4 imply $\tau=I$. Part 3 of Proposition 4 further implies $I=0$. Since CO is trapped between $I$ (since $I$ is locally convex) and 0 , it also agrees with them. Hence Conditions 3 and 1 imply 2. Condition 2 easily implies Condition 1 since $\tau$ is Hausdorff. Hence the six conditions are equivalent.

We remark that if $(L, \tau)$ is compact topological lattice of finite breadth, then $\tau=I$ [5]. Hence all the equivalences of Theorem 5 apply to $(L, \tau)$. It is known that a finite-dimensional compact con- 
nected topological lattice has finite breadth [9].

For complete distributive lattices one obtains a purely algebraic description of lattices which are topological lattices in the interval topology.

THEOREM 6. Let $L$ be a distributive lattice. The following are equivalent:

(1) $L$ is complete and completely distributive.

(2) $L$ is complete and $(L, I)$ is Hausdorff.

(3) $L$ is complete and $L$ can be embedded in a product of unit intervals (under coordinatewise order) by an lattice isomorphism which preserves all joins and all meets.

(4) L admits a topology $\tau$ for which $(L, \tau)$ is a compact topological lattice with enough continuous lattice homomorphisms into the unit interval (with usual order) to separate points.

(5) $L$ admits a topology $\tau$ for which $(L, \tau)$ is a compact topological lattice with a basis of open convex sublattices.

Proof. Theorems 4 and 5 of [6] imply the equivalence of Conditions 4 and 5. Strauss has shown the equivalence of Conditions 1 and 2 [13, Theorem 7] and the implication of Condition 3 by Condition 2 [13, Theorem 6]. It is readily seen that Condition 3 implies that $L$ is a closed subset in the product topology of unit intervals (where the unit internal carries its normal topology); hence $L$ is a compact topological lattice in its relative topolopy. Since a product of intervals has a basis of open convex sublattices, the intersection of this basis with $L$ endows $L$ with such a basis. Hence Condition 3 implies Condition 5. That Condition 5 implies Condition 2 follows from Theorem 5 above.

THEOREM 7. Let $B$ be a Boolean lattice. The following are equivalent:

(1) $B$ is complete and completely distributive.

(2) $B$ admits a topology $\tau$ for which $(B, \tau)$ is a compact topological lattice.

(3) $B$ is isomorphic with the Boolean lattice of subsets of some set.

(4) $B$ is isomorphic to a product of $\{0,1\}$ with $0<1$.

(5) $B$ is complete and $(B, I)$ is Hausdorff.

Proof. By Theorem 6, Conditions 1 and 5 are equivalent and imply Condition 2. Strauss has shown Condition 2 implies Condition 1 [13, Theorem 1].

Tarski has shown that Condition 1 implies Condition 3 (see [14] or [4, p. 119]). If $B$ is isomorphic to all subsets of a set $X$, then it 
can be identified with $\{0,1\}^{x}$ by a lattice isomorphism. Hence Condition 3 implies Condition 4 . Since any product of complete chains is completely distributive [4, p. 120], Condition 4 implies Condition 1 .

3. The convex-order topology. In the preceding section we gave conditions under which a topological lattice had the interval topology and for which all the intrinsic topologies collapsed to this topology. The conditions for a topological lattice to have the order or convex-order topologies are much more general.

THEOREM 8. Let $(L, \tau)$ be a topological lattice with $\tau$ a regular, agreeable topology. If each $x \in L$ has a complete neighborhood, then $\tau=\mathrm{CO}$. ( $A$ subset is complete if every increasing net in the subset has a sup in the subset, and dually).

Proof. By Proposition 3, the CO topology is finer than $\tau$.

Conversely, let $U$ be a basic open convex set in the CO topology. If $U \notin \tau$, then there exists $x$ in $U$ and a net $\left\{x_{\alpha}\right\}$ converging to $x$ in $(L, \tau)$ such that $x_{\alpha} \notin U$ for all $\alpha$.

Let $N$ be a complete neighborhood of $x$ in $\tau$. Let $D$ be the set of all sequences $\left\{W_{n}: n=1,2, \cdots\right\}$ satisfying for all $n$,

(i) $x \in W_{n}^{\circ}, W_{n}=W_{n}^{*} \subset N$

(ii) $\left(W_{n} \vee W_{n}\right) \cup\left(W_{n} \wedge W_{n}\right) \subset W_{n-1}^{\circ}$.

If $\left\{W_{n}\right\},\left\{V_{n}\right\} \in D$, we define $\left\{W_{n}\right\} \geqq\left\{V_{n}\right\}$ if $W_{n} \subset V_{n}$ for all $n$. It is straightforward to verify that $(D, \leqq)$ is a directed set. If $\left\{W_{n}\right\} \in D$, let $W=\cap W_{n}$. Condition (i) implies $x \in W \subset N$ and $W$ is closed. Condition (ii) implies $W$ is a sublattice. Since $\tau$ is agreeable, $N$ is complete, and $W$ is closed, $W$ has a largest element $w^{+}$and a smallest element $w^{-}$.

If $V$ is an closed neighborhood of $x$ contained in $N$, then employing the regularity of $\tau$ and the continuity of $\vee$ and $\wedge$, one can construct $\left\{V_{n}\right\} \in D$ such that $V=V_{1}$. Hence $v^{+} \in \cap V_{n} \subset V$. Thus the net $\left\{w^{+}:\left\{W_{n}\right\} \in D\right\}$ is a monotonic decreasing net which converges to $x$ in the $\tau$-topology. It follows from the continuity of the lattice operations that $\left\{w^{+}\right\} \downarrow x$. Dually $\left\{w^{-}\right\} \uparrow x$. Hence residually many of the $\left\{w^{+}\right\}$and $\left\{w^{-}\right\}$are in $U$. Fix $\left\{W_{n}\right\} \in D$ such that $w^{+}, w^{-} \in U$.

For each $n$, pick $x_{n} \in\left\{x_{\alpha}\right\} \cap W_{n}$. If $m>n$, then

$$
\begin{aligned}
\bigvee_{k=n}^{m} x_{k} & \in \bigvee_{k=n}^{m} W_{k} \subset\left(\bigvee_{k=n}^{m-2} W_{k}\right) \vee W_{m-1} \vee W_{m-1} \\
& \subset\left(\left(_{k=n}^{m-3} W_{k}\right) \vee W_{m-2} \vee W_{m-2} \subset \cdots \subset W_{n-1} .\right.
\end{aligned}
$$

Thus for all $m>n, y_{m}=\bigvee_{k=n}^{m} x_{k} \in W_{n-1}$. Since $W_{n-1} \subset N, W_{n-1}$ is closed, $N$ is complete, and the sequence $y_{m}$ is monotonic increasing, 
there exists $a_{n} \in W_{n-1}$ such that $a_{n}=\sup \left\{x_{k}: k \geqq n\right\}$. The sequence $a_{n}$ is a decreasing sequence contained in $N$, and hence converges to $a=\inf \left\{a_{n}\right\}$. Since the sequence $\left\{a_{n}\right\}$ is eventually in each $W_{n}$ and each $W_{n}$ is closed, we conclude $a \in W=\cap W_{n}$. Hence $a \leqq w^{+}$.

Dually let $b_{n}=\inf \left\{x_{k}: k \geqq n\right\}$ and $b=\sup \left\{b_{n}\right\}$. Then $w^{-} \leqq b$. Since $b_{n} \leqq a_{n}$ for all $n, w^{-} \leqq b \leqq a \leqq w^{+}$. Since $U$ is convex, $a, b \in$ $U$. Since $a_{n} \downarrow a$ and $b_{n} \uparrow b$ and $a, b \in U$, there exists $m$ such that $a_{m}$, $b_{m} \in U$. Since $b_{m} \leqq x_{m} \leqq a_{m}$, we have $x_{m} \in U$. However, this is in contradiction to $x_{m} \in\left\{x_{\alpha}\right\}$ and $x_{\alpha} \notin U$ for all $\alpha$.

The next lemma is a standard and easily proved result about topological lattices (see [7] or [13]).

LEMma 9. Let $K$ be a compact subset of a topological lattice. If $\left\{x_{\alpha}\right\}$ is a monotonically increasing (decreasing) net in $K$, then the net converges to its sup (inf).

THEOREM 10. Let $L$ be a topological lattice which is (i) compact or (ii) locally compact and connected. Then $L$ has the convex order topology.

Proof. If $L$ is compact, it is well known via the work of Nachbin [10] that $L$ is locally convex. This fact together with Lemma 9 implies the topology on $L$ is agreeable and $L$ is complete. The conclusion then follows from Theorem 8.

If $L$ is locally compact and connected, Anderson has shown $L$ is locally convex [1]. Suppose $u_{\alpha} \downarrow x$. Let $U$ be a compact neighborhood of $x$. Since $\left[x, u_{\alpha}\right]=\left(L \wedge u_{\alpha}\right) \vee x$ is connected, if $u_{\alpha}$ is not residually in $U$, then cofinally there exists $y_{\alpha}$ in the boundary of $U$ such that $x \leqq y_{\alpha} \leqq u_{\alpha}$. By compactness of $U$, we can assume by picking subnets if necessary that $\left\{y_{\alpha}\right\}$ converges to some $y$ in the boundary of $U$.

Fix some $\alpha$. If $\beta>\alpha$, then $y_{\beta} \leqq u_{\beta} \leqq u_{\alpha}$. Thus $y_{\beta} \wedge u_{\alpha}=y_{\beta}$ for all $\beta>\alpha$ for which $y_{\beta}$ is defined. Since $y_{\beta} \wedge u_{\alpha}$ converges to $y \wedge$ $u_{\alpha}$, we have $y \wedge u_{\alpha}=y$, i.e., $y \leqq u_{\alpha}$ for all $u_{\alpha}$ not in $U$. Since $x=$ inf $\left\{u_{\alpha}\right\}, y \leqq x$. Similarly, since each $y_{\alpha} \geqq x$, by continuity of $\wedge, y \geqq$ $x$. Hence $y=x$. But this is impossible since $x$ is not in the boundary of $U$. Thus we conclude the topology of $L$ is agreeable. Since $L$ is locally compact, Lemma 9 implies each point has a complete neighborhood. Hence by Theorem $8, L$ has the convex order topology.

It is a consequence of the preceding theorem that a lattice admits at most one topology for which it is a compact (or locally compact connected) topological lattice, namely the convex order topology. This theorem also allows a nice algebraic condition for continuity of homomorphisms between compact (or locally compact connected) topological lattices. It follows that any isomorphism between such lattices is a 
homeomorphism.

Proposition 11. Let $L$ and $K$ be lattices, $f$ a homomorphism from $L$ into K. If $u_{\alpha} \downarrow x\left(t_{\alpha} \uparrow x\right)$ implies $f\left(u_{\alpha}\right) \downarrow f(x)\left(f\left(t_{\alpha}\right) \uparrow f(x)\right)$, then $f$ is continuous if $L$ and $K$ are given the convex order topologies.

Proof. Let $U$ be a basic convex, open set in $K$. Then $f^{-1}(U)$ is convex in $L$. Suppose $x \in f^{-1}(U)$ and $\left\{x_{\alpha}\right\}$ order converges to $x$. Then there exists $u_{\alpha} \downarrow x, t_{\alpha} \uparrow x$ such that for all $\alpha, u_{\alpha} \geqq x_{\alpha} \geqq t_{\alpha}$. Then $f\left(u_{\alpha}\right) \geqq$ $f\left(x_{\alpha}\right) \geqq f\left(t_{\alpha}\right)$ and by hypothesis $f\left(u_{\alpha}\right) \downarrow f(x)$ and $f\left(t_{\alpha}\right) \uparrow f(x)$. Hence since $U$ is open $f\left(x_{\alpha}\right)$ is eventually in $U$. Thus $x_{\alpha}$ is eventually in $f^{-1}(U)$. Hence $f^{-1}(U)$ is open and $f$ is continuous.

It is shown in [13] that if $(L, \tau)$ is a topological lattice for which $\tau$ is a first countable regular topology for which every point has a $\sigma$ complete neighborhood, then $\tau$ is finer than the order topology. If further, $\tau$ is agreeable, Propositions 2 and 3 show $\tau$ is the order topology. Since in the proof of Theorem 10, it was shown that the topology of a locally compact connected or a compact topological lattice is agreeable, it follows that

THEOREM 12. Let $L$ be a compact or locally compact connected topological lattice which is metrizable. Then $L$ has the order topology.

The theorem for the compact case appears in [7] and [13]. It is not known whether the theorem remains true without metrizability.

4. Compact semilattices. In this section we give an internal characterization of the topology of a compact semilattice. If $S$ is a semilattice we say $I$ is an ideal of $S$ if $L(I)=I$. If $A$ is an ideal in $S$, define $A^{+}$by $x \in A^{+}$if there exists a net $x_{\alpha}$ in $A$ such that $x_{\alpha} \uparrow x$.

THEOREM 13. Let $S$ be a compact topological semilattice. An ideal $A$ of $S$ is closed if and only if $A=A^{+}$.

Proof. Suppose $A$ is closed. If $x \in A$, then the constant net $x$ is a monotonic increasing net increasing to $x$. Hence $A \subset A^{+}$. If $x_{\alpha}$ is a net in $A$ and $x_{\alpha} \uparrow x$, then $x_{\alpha}$ converges to $x$ in the topology of $S$ (a monotonically increasing net converges to its sup in a compact topological semilattice). Hence $x \in A$. Thus $A=A^{+}$.

Conversely let $A=A^{+}$. Let $y \in A^{*}$. Let $D$ be the set of all sequences $\left\{W_{n}: n=1,2, \cdots,\right\}$ satisfying for all $n$,

(i) $x \in W_{n}^{\circ}, W_{n}=W_{n}^{*}$

(ii) $W_{n} \wedge W_{n} \subset W_{n-1}^{\circ}$. 
If $\left\{W_{n}\right\},\left\{V_{n}\right\} \in D$, we define $\left\{W_{n}\right\} \geqq\left\{V_{n}\right\}$ if $W_{n} \subset V_{n}$ for all $n$. Then $(D, \leqq)$ is a directed set. If $\left\{W_{n}\right\} \in D$, let $W=\cap W_{n}$. Then $W$ is closed and is a subsemilattice. Hence $W$ has a minimal element $w^{-}$. As in the proof of Theorem 8, $\left\{w^{-}:\left\{W_{n}\right\} \in D\right\}$ is a monotonically increasing net and $w^{-\uparrow} y$.

Fix a specific $w^{-}$associated with a $\left\{W_{n}\right\}$. Since $y \in A^{*}$, for each $n$ there exists $b_{n} \in W_{n} \cap A$. Let $\partial_{n}=\Lambda_{m>n} b_{m}$. Then $\partial_{n}$ is an increasing sequence, each $\partial_{n} \in A$ since $A$ is an ideal, and as in the proof of Theorem 8, $\partial_{n} \uparrow \partial \in W$. Since $A=A^{+}, \partial \in A$. Since $w^{-} \leqq \partial$ and $A$ is an ideal, $w^{-} \in A$. But since the net $\left\{w^{-}\right\} \uparrow y$, we conclude $y \in A$. Hence $A$ is closed.

Theorem 13 makes possible an algebraic description of the closure of an ideal in a compact topological semilattice.

CoRollary 14. Let I be an ideal of a compact topological semilattice $S$. Then $I^{*}=I^{++}$.

Proof. Since $I \subset I^{*}$, we have $I^{+} \subset\left(I^{*}\right)^{+}$. By Theorem 13, $\left(I^{*}\right)^{+}=$ $I^{*}$. Hence $I^{+} \subset I^{*}$. A repetition of the argument with $I^{+}$replacing $I$ shows $I^{++} \subset I^{*}$.

Let $y \in I^{+}$and $x \leqq y$. Then there exists a net $\left\{y_{\alpha}\right\}$ in $I$ such that $y_{\alpha} \uparrow y$. Then $x \wedge y_{\alpha} \uparrow x$ and $x \wedge y_{\alpha} \in I$ for all $\alpha$. Thus $x \in I^{+}$; hence we have shown $I^{+}$is an ideal. It is essentially shown in the proof of Theorem 13 that if $y \in I^{*}$, then $y \in\left(L\left(I^{+}\right)\right)^{+}$. Since $I^{+}$is an ideal $L\left(I^{+}\right)=I^{+}$. Thus $y \in I^{++}$. Hence $I^{++}=I^{*}$.

A principal application of Theorem 13 is an algebraic or intrinsic method of defining the topology of a compact topological semilattice. It is known that if $S$ is a compact topological semilattice, then the space of all closed ideals $S^{\prime}$ of $S$ ordered by inclusion and considered as a subspaces of $2^{S}$ is a compact distributive topological lattice; furthemore the mapping sending $s$ into $L(s)$ is a topological isomorphism from $S$ into $S^{\prime}$ (see e.g. [8, Theorem 1.2]). Since the closed ideals of $S$ can be identified algebraically as those ideals for which $I=I^{+}$and since the topology of $S^{\prime}$ can be defined algebraically as the convex-order topology (Theorem 10), the topology of $S$ is determined by its algebraic structure.

THEOREM 15. Let $f$ be a homomorphism from a compact topological semilattice $S$ onto a compact topological semilattice $T$. If $f$ has the property that for $x_{\alpha} \uparrow x, f\left(x_{\alpha}\right) \uparrow f(x)$ and for $y_{\alpha} \downarrow y, f\left(y_{\alpha}\right) \downarrow f(y)$, then $f$ is continuous.

The proof of this theorem breaks down conveniently into several steps. 
(i) If $t \in T, f^{-1}(t)$ has a least element. Since $f$ is a homomorphism $f^{-1}(t)$ is a semilattice. Hence it is a monotonically decreasing net indexed by itself. Since $S$ is compact, the net monotonically decreases to some $s$. Hence by hypothesis $f(s)=t$. Thus $s$ is a least element for $f^{-1}(t)$.

(ii) If $A$ is an ideal, $f(A)^{+}=f\left(A^{+}\right)$. Suppose $y \in f(A)^{+}$. Then there exists a net $y_{\alpha} \uparrow y$ where $y_{\alpha} \in f(A)$ for all $\alpha$. There exists $w_{\alpha} \in$ $A$ such that $f\left(w_{\alpha}\right)=y_{\alpha}$ for each $\alpha$. There exists $x_{\alpha}$, the least element of $f^{-1}\left(y_{\alpha}\right)$; hence $x_{\alpha} \leqq w_{\alpha}$. Since $A$ is an ideal, $x_{\alpha} \in A$. If $\alpha \leqq \beta$, then $f\left(x_{\alpha} \wedge x_{\beta}\right)=f\left(x_{\alpha}\right) \wedge f\left(x_{\beta}\right)=y_{\alpha} \wedge y_{\beta}=y_{\alpha}$; hence $x_{\alpha} \wedge x_{\beta} \in f^{-1}\left(y_{\alpha}\right)$. Since $x_{\alpha}$ is the least element of $f^{-1}\left(y_{\alpha}\right), x_{\alpha}=x_{\alpha} \wedge x_{\beta}$. Hence the net $x_{\alpha}$ is increasing. Since $S$ is compact, $x_{\alpha} \uparrow x$ for some $x \in A^{+}$. By hypothesis $f\left(x_{\alpha}\right) \uparrow f(x)$, i.e., $y_{\alpha} \uparrow f(x)$. Thus $p=f(x) \in f\left(A^{+}\right)$. Conversely, let $t=f(s) \in f\left(A^{+}\right)$. Then there exists a net $s_{\alpha} \uparrow s, s_{\alpha} \in A$ for each $\alpha$. By hypothesis $f\left(s_{\alpha}\right) \uparrow f(s)$. Hence $t \in f(A)^{+}$. Thus $f(A)^{+}=f\left(A^{+}\right)$.

(iii) $f$ induces a homomorphism $f^{\prime}: S^{\prime} \rightarrow T^{\prime}$, the lattices of closed ideals of $S$ and $T$ resp. If $A$ is a closed ideal of $S$, define $f^{\prime}(A)$ to be $f(A)$. Since $f$ is onto, $f(A)$ is an ideal. Also $f(A)^{+}=f\left(A^{+}\right)=f(A)$; hence $f(A)$ is closed, i.e., $f^{\prime}(A) \in T^{\prime}$. Always $f(A \cup B)=f(A) \cup f(B)$ and $f(A \cap B) \subset f(A) \cap f(B)$. Suppose $t \in f(A) \cap f(B)$; then there exists $a \in A, b \in B$ such that $f(a)=t=f(b)$. Let $x$ be the least element of $f^{-1}(t)$; then $x \leqq a, x \leqq b$. If $A$ and $B$ are ideals, then $x \in A \cap B$. Hence $t=f(x) \in f(A \cap B)$. Thus $f(A \cap B)=f(A) \cap f(B)$.

(iv) $f^{\prime}$ preserves limits of increasing and decreasing nets.

In $S^{\prime}$ and $T^{\prime}$ the limit of a decreasing net is just the intersection. An argument similar to the one just given to show $f^{\prime}$ preserves finite intersections will show $f^{\prime}$ also preserves arbitrary intersections. If $\left\{A_{\alpha}\right\}$ is an increasing net in $S^{\prime}$, then the limit is $\left(\cup A_{\alpha}\right)^{*}$ and the limit of $f\left(A_{\alpha}\right)$ is $\left.\left(\cup f A_{\alpha}\right)\right)^{*}$. Now $\left.f\left(\left(\cup A_{\alpha}\right)^{*}\right)=f\left(\left(\cup A_{\alpha}\right)^{++}\right)=\left(f \cup A_{\alpha}\right)\right)^{++}$ (by two applications of (ii)) $=\left(\cup f\left(A_{\alpha}\right)\right)^{++}=\left(\cup f\left(A_{\alpha}\right)\right)^{*}$. Hence $f^{\prime}$ preserves limits.

(v) The homomorphism $f$ is continuous. Theorems 10 and 11 imply that $f^{\prime}$ is continuous. Since $S$ and $T$ are embedded in $S^{\prime}$ and $T^{\prime}, f^{\prime}$ restricted to their images is continuous. But this restriction of $f^{\prime}$ is just $f$.

CoROLLARY 16. Let $h$ be an isomorphism from a compact topological semilattice $S$ onto a compact topological semilattice $T$. Then $h$ is a homeomorphism. Hence a fixed semilattice admits at most one topology for which it is a compact topological semilattice.

Proof. Clearly $h$ and $h^{-1}$ preserve limits of increasing and decreasing nets. Hence the conclusion follows from Theorem 15 .

For any two compact topologies, the identity mapping must be a 
homeomorphism. Hence the two agree.

Anderson and Hunter [2] have studied some classes of groups and semigroups in which each automorphism is continuous; this property they call van der Waerden property. Corollary 16 shows that compact semilattices are such semigroups.

\section{REFERENCES}

1. L. W. Anderson, One dimensional topological lattices, Proc. Amer. Math. Soc., 10 (1959), 327-333.

2. L. W. Anderson and R. P. Hunter. Homomorphisms of compact semigroups, Duke Math. J., 38 (1971), 409-414.

3. K. Atsumi, On complete lattices having the Hausdorff interval topology, Proc. Amer. Math. Soc., 17 (1966), 197-199.

4. G. Birkhoff, Lattice theory, Amer. Math. Soc. Colloquium Publications, 3rd ed., vol. XXV, Amer. Math. Soc., Providence, R. I., 1967.

5. T. H. Choe, Intrinsic topologies in a topological lattice, Pacific J. Math., 28 (1969), 49-52.

6. E. B. Davies, The existence of characters on topological lattices, J. London Math. Soc., 43 (1968), 217-220.

7. J. D. Lawson, Vietoris mappings and embeddings of topological semilattices, University of Tennessee dissertation, 1967.

8. ㄴ, Lattices with no interval homomorphisms, Pacific J. Math., 32 (1970), 459465.

9. - The relation of breadth and codimension in topological semilattices, Duke Math. J., 37 (1970), 207-212.

10. L. Nachbin, Topology and Order, D. Van Nostrand Co., Inc., Princeton, N. J., 1965.

11. B. C. Rennie, The Theory of Lattices, Foister and Jagg, Cambridge, England, 1952.

12. - Lattices, Proc. London Math. Soc., 52 (1951), 386-400.

13. D. P. Strauss, Topological lattices, Proc. London Math. Soc., 18 (1968), 217-230.

14. A. Tarski, Sur les classes closes par rapport à certaines opérations élémentaires, Fund. Math., 16 (1929), 195-197.

15. A. J. Ward, On relations between certain intrinsic topologies in certain partially ordered sets, Proc., Cambridge Philos. Soc. 51 (1955), 254-261.

Received October 11, 1971. The research for this paper was supported by NSF grant GP-25014.

LOUISIANA STATE UNIVERSITY 


\section{PACIFIC JOURNAL OF MATHEMATICS}

\section{EDITORS}

\section{H. SAMELSON}

Stanford University

Stanford, California 94305

C. R. Новву

University of Washington Seattle, Washington 98105

\section{J. DuGundJI}

Department of Mathematics University of Southern California Los Angeles, California 90007

RICHARD ARENS

University of California Los Angeles, California 90024

\section{ASSOCIATE EDITORS}
E. F. BECKENBACH
B. H. NeumanN
F. WOLF
K. YoSHIDA

\section{SUPPORTING INSTITUTIONS}

\author{
UNIVERSITY OF BRITISH COLUMBIA \\ CALIFORNIA INSTITUTE OF TECHNOLOGY \\ UNIVERSITY OF CALIFORNIA \\ MONTANA STATE UNIVERSITY \\ UNIVERSITY OF NEVADA \\ NEW MEXICO STATE UNIVERSITY \\ OREGON STATE UNIVERSITY \\ UNIVERSITY OF OREGON \\ OSAKA UNIVERSITY
}

\author{
UNIVERSITY OF SOUTHERN CALIFORNIA \\ STANFORD UNIVERSITY \\ UNIVERSITY OF TOKYO \\ UNIVERSITY OF UTAH \\ WASHINGTON STATE UNIVERSITY \\ UNIVERSITY OF WASHINGTON \\ $*{ }^{*}$
AMERICAN MATHEMATICAL SOCIETY
NAVAL WEAPONS CENTER
}

The Supporting Institutions listed above contribute to the cost of publication of this Journal, but they are not owners or publishers and have no responsibility for its content or policies.

Mathematical papers intended for publication in the Pacific Journal of Mathematics should be in typed form or offset-reproduced, (not dittoed), double spaced with large margins. Underline Greek letters in red, German in green, and script in blue. The first paragraph or two must be capable of being used separately as a synopsis of the entire paper. The editorial "we" must not be used in the synopsis, and items of the bibliography should not be cited there unless absolutely necessary, in which case they must be identified by author and Journal, rather than by item number. Manuscripts, in duplicate if possible, may be sent to any one of the four editors. Please classify according to the scheme of Math. Rev. Index to Vol, 39. All other communications to the editors should be addressed to the managing editor, Richard Arens, University of California, Los Angeles, California, 90024.

50 reprints are provided free for each article; additional copies may be obtained at cost in multiples of 50 .

The Pacific Journal of Mathematics is issued monthly as of January 1966. Regular subscription rate: $\$ 48.00$ a year (6 Vols., 12 issues). Special rate: $\$ 24.00$ a year to individual members of supporting institutions.

Subscriptions, orders for back numbers, and changes of address should be sent to Pacific Journal of Mathematics, 103 Highland Boulevard, Berkeley, California, 94708.

PUBLISHED BY PACIFIC JOURNAL OF MATHEMATICS, A NON-PROFIT CORPORATION

Printed at Kokusai Bunken Insatsusha (International Academic Printing Co., Ltd.), 270, 3-chome Totsuka-cho, Shinjuku-ku, Tokyo 160, Japan. 


\section{Pacific Journal of Mathematics}

\section{Vol. 44, No. $2 \quad$ June, 1973}

Tsuyoshi Andô, Closed range theorems for convex sets and linear liftings . . . . . . 393

Richard David Bourgin, Conically bounded sets in Banach spaces . . . . . . . . . 411

Robert Jay Buck, Hausdorff dimensions for compact sets in $R^{n} \ldots \ldots \ldots \ldots \ldots \ldots . \ldots 421$

Henry Cheng, A constructive Riemann mapping theorem ................ 435

David Fleming Dawson, Summability of subsequences and stretchings of

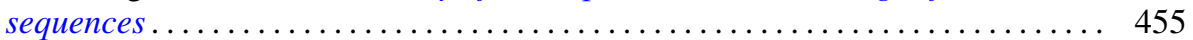

William Thomas Eaton, A two sided approximation theorem for 2-spheres ....... 461

Jay Paul Fillmore and John Herman Scheuneman, Fundamental groups of compact complete locally affine complex surfaces ....................... 487

Avner Friedman, Bounded entire solutions of elliptic equations . . . . . . . . . . . 497

Ronald Francis Gariepy, Multiplicity and the area of an $(n-1)$ continuous

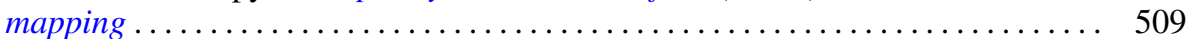

Andrew M. W. Glass, Archimedean extensions of directed interpolation groups . . . . 515

Morisuke Hasumi, Extreme points and unicity of extremum problems in $H^{1}$ on

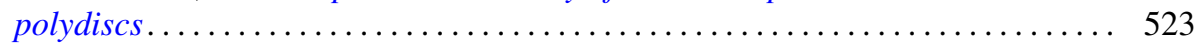

Trevor Ongley Hawkes, On the Fitting length of a soluble linear group . . . . . . 537

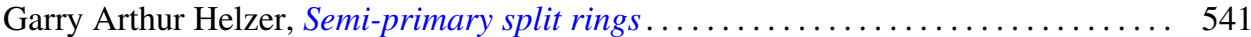

Melvin Hochster, Expanded radical ideals and semiregular ideals . . . . . . . . . 553

Keizō Kikuchi, Starlike and convex mappings in several complex variables . . . . . . 569

Charles Philip Lanski, On the relationship of a ring and the subring generated by its

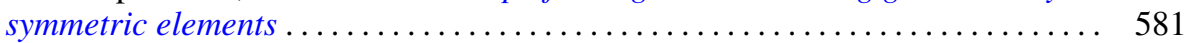

Jimmie Don Lawson, Intrinsic topologies in topological lattices and semilattices ........................................... 593

Roy Bruce Levow, Counterexamples to conjectures of Ryser and de Oliveira ...... 603

Arthur Larry Lieberman, Some representations of the automorphism group of an infinite continuous homogeneous measure algebra ..........

William George McArthur, $G_{\delta}$-diagonals and metrization theorems $\ldots .$.

James Murdoch McPherson, Wild arcs in three-space. II. An invariant of

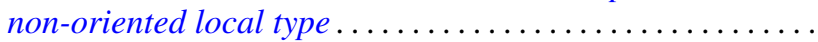

H. Millington and Maurice Sion, Inverse systems of group-valued measures ...

C. Edward Moore, Concrete semispaces and lexicographic separation of convex

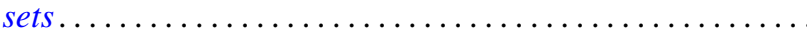

Jingyal Pak, Actions of torus $T^{n}$ on $(n+1)$-manifolds $M^{n+1}$.

Merrell Lee Patrick, Extensions of inequalities of the Laguerre and Turán type . . . . 675

Harold L. Peterson, Jr., Discontinuous characters and subgroups of finite index. . . . 683

S. P. Philipp, Abel summability of conjugate integrals . . . . . . . . . . . . . 693

R. B. Quintana and Charles R. B. Wright, On groups of exponent four satisfying an

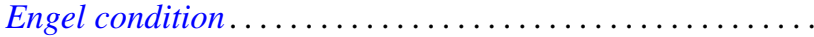

Marlon C. Rayburn, On Hausdorff compactifications. . . . . . . . . .

Martin G. Ribe, Necessary convexity conditions for the Hahn-Banach theorem in

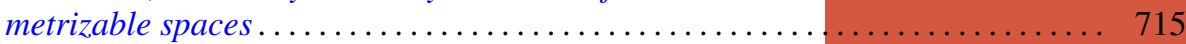

Ryōtarō Satō, On decomposition of transformations in infinite measure spaces .... 733

Peter Drummond Taylor, Subgradients of a convex function obtained from a

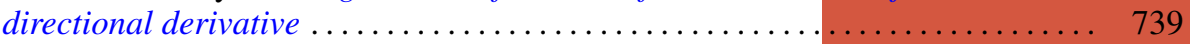

James William Thomas, A bifurcation theorem for $k$-set contractions . . . . . . . . 749 Clifford Edward Weil, A topological lemma and applications to real functions . . . . 757

Stephen Andrew Williams, A nonlinear elliptic boundary value problem . . ....... 767

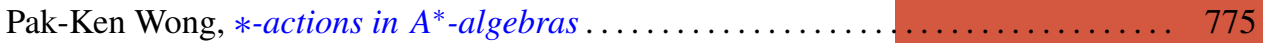

